\title{
New Families of Isospectral Hydrogen-like Potentials
}

\author{
J. Oscar Rosas-Ortiz* \\ Departamento de Física Teórica ${ }^{\dagger}$ \\ Universidad de Valladolid, 47011 Valladolid, Spain
}

August 19, 2018

\begin{abstract}
By applying algebraic techniques, we construct a two-parametric family of strictly isospectral Hydrogen-like potentials, as well as some of its one-parametric limits. An additional one-parametric almost isospectral family of Hydrogen-like potentials is also investigated. It is argued that the construction of a SUSY partner Hamiltonian using a factorization energy $\delta$ less than the ground state energy of the departure Hamiltonian is unnecessarily restrictive.
\end{abstract}

Key-Words: Factorization, Hydrogen atom

PACS: 03.65.Ge, 03.65.Fd, 03.65.Ca

${ }^{*}$ On leave of absence from Departamento de Física, CINVESTAV-IPN, A.P. 14-740, 07000 México D.F., Mexico. E-mail orosas@fis.cinvestav.mx

${ }_{\dagger}^{\dagger}$ electronic address:orosas@klander.fam.cie.uva.es 
There now exists a considerable amount of work relating to exactly solvable onedimensional potentials in Quantum Mechanics (QM). The main interest has been to enlarge the number of analitically solvable potentials using diverse techniques: the Darboux transformation [1], the Gelfand-Levitan formalism [2], the standard and modified factorizations [3-4], the supersymmetric Quantum Mechanics (SUSY QM) [5], etc. The underlying ideas of most of these procedures have been summarized in an algebraic scheme in which a first order differential operator intertwines two different Hamiltonians [6]. In particular, the generation of one-parametric families of potentials isospectral to the traditional ones [2-9] can be performed by means of this first order intertwinning technique. This method leads also in a natural way to the factorization of the implied Hamiltonians.

Recently, a generalization of this technique, in which the intertwinning operator is of second order, has been used to derive a two-parametric family of potentials isospectral to the harmonic oscillator [10]. This second order intertwinning technique (SOIT) is a particular case with $n=2$ of the $n$-order intertwinning technique which appears to be the best way to introduce the higher order SUSY Quantum Mechanics [11]. In principle, the latter makes possible to generate $n$-parametric families of Hamiltonians isospectral to a given Hamiltonian [12, 13].

In this paper we will generate a 2-parametric family of radial isospectral Hydrogen-like potentials by means of the SOIT. We shall show also that the one-parametric family of potentials derived by Fernández [8] can be recovered from ours. As a final result, we will find a 1-parametric family of potentials having the same energy levels as the corresponding radial Hydrogen-like potentials except for the ground state energy level.

The standard procedure to deal with Hydrogen-like potentials in QM reduces to solve the eigenproblem for a particle in a 1-dimensional effective potential $V_{l}(r)=l(l+1) / r^{2}-$ $2 / r$, where $l=0,1,2, \ldots$ is the azimuthal quantum number and $r$ is a dimensionless radial coordinate. By simplicity, instead of working with the standard radial wavefunctions $R(r)$, we will work with the functions $\psi(r) \equiv r R(r)$ with an inner product defined by $\left\langle\psi, \psi^{\prime}\right\rangle \equiv$ $4 \pi \int_{0}^{+\infty} \bar{\psi}(r) \psi^{\prime}(r) d r$. As it is well known, the eigenvalues of the radial Hamiltonian $H_{l}=$ $-d^{2} / d r^{2}+V_{l}(r)$ (with fixed $l$ ) are given by:

$$
E_{n} \equiv E_{l k}=-\frac{1}{(l+k)^{2}} ; \quad k=1,2,3, \ldots
$$

where $l+k=n$.

We are looking for a Hamiltonian $\widetilde{H}_{l^{\prime}}=-d^{2} / d r^{2}+\widetilde{V}_{l^{\prime}}(r)$, such that the following intertwinning relationship is satisfied [6]:

$$
\widetilde{H}_{l^{\prime}} A=A H_{l},
$$


where $A$ is a second order differential operator to be determined

$$
A \equiv \frac{d^{2}}{d r^{2}}+\beta(r) \frac{d}{d r}+\gamma(r)
$$

Equations (2-3) and the explicit form of $H_{l}$ and $\widetilde{H}_{l^{\prime}}$ lead to the following relations between $V_{l}(r), \tilde{V}_{l^{\prime}}(r), \beta(r)$ and $\gamma(r)$ :

$$
\begin{array}{r}
\beta \beta^{\prime \prime}-\frac{\beta^{2}}{2}+\left(2 \gamma(r)-\beta^{\prime}-\frac{\beta^{2}}{2}\right) \beta^{2}+2 c=0, \\
\widetilde{V}_{l^{\prime}}(r)=\frac{l(l+1)}{r^{2}}-\frac{2}{r}+2 \beta^{\prime}, \\
2 \gamma(r)=\beta^{2}-\beta^{\prime}-2 \frac{l(l+1)}{r^{2}}+\frac{1}{r}-d,
\end{array}
$$

where $c$ and $d$ are, in principle, arbitrary constants and the prime denotes derivative with respect to $r$. The key point becomes now to solve the non-linear second order differential equation (4) for $\beta(r)$. Let us first enforce that the operator $A$ includes $a_{l-1} a_{l}$ as a particular case, where $a_{l}=(-d / d r+l / r-1 / l)$ is the standard factorization operator for the radial Hydrogen-like Hamiltonians [3]. This condition permit us, without a loss of generality, to fix the constants $c$ and $d$ in (4-6) as

$$
c=\frac{(2 l-1)^{2}}{4 l^{4}(l-1)^{4}}, \quad d=\frac{1+(2 l-1)^{2}}{2 l^{2}(l-1)^{2}} .
$$

After including (7) in (4-6), it turns out that the general solution to (4) becomes:

$$
\begin{gathered}
\beta(r)=\frac{1-2 l}{l^{2}(l-1)^{2}}\left[\frac{d}{d r} \log \left(\frac{g_{2}(r)}{g_{1}(r)}\right)\right]^{-1}, \quad l=2,3, \ldots, \\
g_{1}(r)=\left\{1-\frac{\nu_{1}}{(2 l) !}\left(\frac{2}{l}\right)^{2 l+1} \int_{0}^{r} x^{2 l} e^{-2 x / l} d x\right\}, \\
g_{2}(r)=e^{r / l(l-1)}\left[1-\frac{r}{l(l-1)}\right]\left\{1+\frac{\nu_{2}}{(2 l-1) !}\left(\frac{2}{l-1}\right)^{2 l-1} \int_{0}^{r} \frac{x^{2 l} e^{-2 x /(l-1)}}{[l(l-1)-x]^{2}} d x\right\},
\end{gathered}
$$

where $\nu_{1}$ and $\nu_{2}$ are integration constants.

The asymptotic behaviour of $\beta(r)$ is given by $\beta(r) \sim(1-2 l) / l(l-1)$, while it diverges as $(2 l-1) / r$, when $r \rightarrow 0^{+}$. This suggests to us to write the new potential $\widetilde{V}_{l^{\prime}}(r)$ in $(5)$ as

$$
\widetilde{V}_{l-2}(r)=V_{l-2}(r)+2 \alpha^{\prime}(r), \quad l=2,3, \ldots
$$

where $l^{\prime}=l-2$ and $\alpha(r) \equiv \beta(r)+(1-2 l) / r$, is an appropriate function that makes evident the limit $\widetilde{V}_{l-2}(r) \rightarrow V_{l-2}(r)$, when $r \rightarrow+\infty$ and $r \rightarrow 0$. The parameter domain 
for which $\alpha^{\prime}(r)$ is free of singularities is given by $\nu_{1}, \nu_{2} \in(-\infty, 1)$; inside this parameter region, the new two-parametric family of potentials $\widetilde{V}_{l-2}(r)$ has the same singularities as $V_{l-2}(r)$. Furthermore, for $\nu_{1}=\nu_{2}=0$ we have $\alpha^{\prime}=0$ and $\widetilde{V}_{l-2}(r)=V_{l-2}(r)$. This means that $V_{l-2}(r)$ not only governs the asymptotic behaviour of $\widetilde{V}_{l-2}(r)$ but it is a member of the family (11).

Now, from equation (2) it becomes apparent that the operator $A$ acting on the eigenfunctions $\left\{\psi_{n l}(r)\right\}$ of $H_{l}$ provides eigenfunctions $\widetilde{\psi}_{n, l-2}(r) \propto A \psi_{n l}(r), l=2,3, \ldots$ of $\widetilde{H}_{l-2}$ with eigenvalues $\widetilde{E}_{l k}=E_{l k}$, i.e.:

$$
\widetilde{\psi}_{n, l-2}(r)=\frac{l(l-1) n^{2}}{\sqrt{\left(n^{2}-l^{2}\right)\left(n^{2}-l^{2}+2 l-1\right)}} A \psi_{n l}(r) .
$$

However, the set $\left\{\widetilde{\psi}_{n, l-2}(r)\right\}$ is not yet complete in the Hilbert space of squareintegrable functions $\mathcal{H}$. This is clear if we try to answer the following questions: are there functions $\widetilde{\psi}_{l-2, \epsilon}(r)$ orthogonal to all the $\widetilde{\psi}_{n, l-2}(r)$ ? If so, are they eigenfunctions of $\widetilde{H}_{l-2}$ ? In order to answer these questions, let us assume that the set $\left\{\widetilde{\psi}_{l-2, \epsilon}\right\}$ exists, then

$$
\left\langle\widetilde{\psi}_{l-2, \epsilon}(r), \widetilde{\psi}_{n, l-2}(r)\right\rangle \propto\left\langle\widetilde{\psi}_{l-2, \epsilon}(r), A \psi_{n l}(r)\right\rangle=\left\langle A^{\dagger} \widetilde{\psi}_{l-2, \epsilon}(r), \psi_{n l}(r)\right\rangle=0
$$

Due to the fact that $\left\{\psi_{n l}(r)\right\}$ is a complete set in $\mathcal{H}$, the kernel of the second order differential operator $A^{\dagger}$ is a 2-dimensional subspace $\mathcal{H}_{\epsilon}$ orthogonal to all the $\widetilde{\psi}_{n, l-2}(r)$, $l=2,3, \ldots$ Let us write them as $\widetilde{\psi}_{l-2, \epsilon}(r)=c_{0} \exp \left[\int f(x) d x\right]$, where $c_{0}$ is a constant and $f(x)$ is to be determined. The equation $A^{\dagger} \widetilde{\psi}_{l-2, \epsilon}(r)=0$ can be rewritten as:

$$
f^{\prime}(r)-\beta(r) f(r)+f^{2}(r)-\beta^{\prime}(r)+\gamma(r)=0 .
$$

This Riccati type differential equation has a general solution given by

$$
f(r)=\frac{1}{l}-\frac{l}{r}+\beta(r)+\frac{d}{d r} \ln \left[c_{1} g_{1}(r)+c_{2} g_{2}(r)\right]
$$

where $g_{1}(r)$ and $g_{2}(r)$ are given in (9-10), and $c_{1}$ and $c_{2}$ are constants. The generic kernel element is given by $\widetilde{\psi}_{l-2, \epsilon}(r)=C_{0} \widetilde{\psi}_{l-2,0}(r)+C_{-1} \widetilde{\psi}_{l-2,-1}(r)$, where

$$
\widetilde{\psi}_{l-2,0}(r)=\sqrt{\left(\frac{1-\nu_{1}}{(2 l) !}\right)\left(\frac{2}{l}\right)^{2 l+1}(2 l-1)}\left(\frac{1}{l(l-1)}\right) \frac{r^{l} e^{-r / l} g_{2}(r)}{W\left(g_{1}, g_{2}\right)}, \quad l=2,3, \ldots,
$$

and

$$
\widetilde{\psi}_{l-2,-1}(r)=\sqrt{\left(\frac{1-\nu_{2}}{2 l(2 l) !}\right)\left(\frac{2}{l-1}\right)^{2 l+1}(2 l-1)}\left(\frac{1}{l(l-1)}\right) \frac{r^{l} e^{-r / l} g_{1}(r)}{W\left(g_{2}, g_{1}\right)}, \quad l=2,3, \ldots,
$$


are both eigenfunctions of $\widetilde{H}_{l-2}$ with eigenvalues $\widetilde{E}_{l-2,0}=-1 / l^{2}, \widetilde{E}_{l-2,-1}=-1 /(l-1)^{2}$, $l=2,3, \ldots$ respectively. In $(15-16), W\left(g_{1}, g_{2}\right)=g_{1}^{\prime}(r) g_{2}(r)-g_{1}(r) g_{2}^{\prime}(r)$ represents the Wronskian of $g_{1}$ and $g_{2}$.

Notice that $\left\{\widetilde{\psi}_{l-2,-1}, \widetilde{\psi}_{l-2,0}, \widetilde{\psi}_{n, l-2}, l=2,3, \ldots\right\}$ is now a complete set in $\mathcal{H}$, and their elements are eigenfunctions of $\widetilde{H}_{l-2}, l=2,3, \ldots$, with eigenvalues:

$$
\widetilde{E}_{l-2, k}=-\frac{1}{(l-1)^{2}},-\frac{1}{l^{2}},-\frac{1}{(l+k)^{2}} ; \quad k=1,2,3, \ldots
$$

Comparing with (1), one can see that this spectrum is identical to that of $\mathrm{H}_{l-2}$, i.e., $E_{l-2, k}=\widetilde{E}_{l-2, k}$. Hence, the Hamiltonian $\widetilde{H}_{l-2}$ is strictly isospectral to $H_{l-2}$, and because $\widetilde{V}_{l-2}(r)$ depends of two free parameters $\nu_{1}$ and $\nu_{2}$, a new two-parametric family of isospectral Hydrogen-like potentials has been generated. Some particular cases are worth discussing in more detail.

Firstly, for $\nu_{1}=\nu_{2}=0$, the well known Hydrogen-like potential $V_{l-2}(r)$ is always recovered because it is a member of the family $\widetilde{V}_{l-2}(r)$.

Let us now to take $\nu_{1}=0\left(\nu_{2}=0\right)$, in this case the 2-parametric family $\widetilde{V}_{l-2}(r)$ is a new 1-parametric family which becomes to a Hydrogen-like potential when $\nu_{2} \rightarrow 0\left(\nu_{1} \rightarrow 0\right)$. In particular, let $\nu_{1}=0$, and

$$
\nu_{2}=\left(\frac{l-1}{2}\right)^{2 l-1} \frac{(2 l-2) !}{\gamma_{l-1}} .
$$

In this case, the function $\alpha(r)$ becomes

$$
\alpha(r)=\frac{1-2 l}{l(l-1)}+\frac{r^{2 l-2} e^{-2 r /(l-1)}}{\gamma_{l-1}-\int_{0}^{r} x^{2 l-2} e^{-2 x /(l-1)} d x}, \quad l=2,3, . .
$$

If we change in (11) $l$ by $l+1$ with $\alpha(r)$ as it is defined above, we get the following one-parametric family of potentials:

$$
\widetilde{V}_{l-1}(r)=V_{l-1}(r)+2 \frac{d}{d r}\left\{\frac{r^{2 l} e^{-2 r / l}}{\gamma_{l}-\int_{0}^{r} x^{2 l} e^{-2 x / l} d x}\right\}, \quad l=1,2, \ldots
$$

which was generated by Fernández in 1984 [8]. Notice that, in the case when $\gamma_{l} \rightarrow 1 / 4$, the family (18) gives the particular case derived by Abraham and Moses [7]. Moreover, when $\gamma_{l} \rightarrow \infty\left(\nu_{2} \rightarrow 0\right)$, we have $\widetilde{V}_{l-1}(r) \rightarrow V_{l-1}(r)$, just as we have proposed.

The previous potentials can be seen as deformations of $V_{l-1}(r)$ induced by the second term in (18), which does not change the behaviour of $V_{l-1}(r)$ at the ends of the interval $[0, \infty)$, but can produce important modifications inside. In particular, there is the possibility of creating one additional well in $V_{l-1}(r)$ whose depth and position can be changed 
by varying $\gamma_{l}$. On the other hand, the two-parametric family of potentials (11) admits also the previous interpretation, but more freedom is given by the deforming term (it depends on two parameters instead of just one as in the previous case). Hence, we get the possibility of introducing now two wells, one of them with its minimum placed around the global minimum of $V_{l-2}(r)$ and the other one pushed further out. As in the previous one-parametric case, the depths and positions of the two wells can be modified by changing the two parameters $\nu_{1}$ and $\nu_{2}$. This is illustrated in Figure 1, where two members of the family $\widetilde{V}_{1}(r)$ of (11) are depicted, together with the undeformed potential $V_{1}(r)$ (dashed line). We have plotted also in Figure 2 the corresponding probability densities for the two energy levels $\widetilde{E}_{1,-1}=-1 / 4, \widetilde{E}_{1,0}=-1 / 9$ with $\nu_{1}=\nu_{2}=-10$. For the lowest level the probability has a maximum around the left well. On the other hand, the first excited state has two maxima, the highest one centered around the right well, while the lowest one is situated around the left well. When we go over the higher excited states, the probabilities resemble more and more the corresponding Hydrogen-like densities.

Up to now, departing from the Hydrogen-like potential $V_{l}(r)$ we have generated a twoparametric family of solvable potentials $\widetilde{V}_{l-2}(r)$, with the same spectrum and singularities as $V_{l-2}(r)$. Now, as the intertwinning operator $A$ is of second order, it is interesting to look for its possible factorization in terms of two first order differential operators $b_{1}$, and $b_{2}$, i.e.:

$$
A=b_{2} b_{1}, \quad b_{j}=\frac{d}{d r}+w_{j}(r), \quad j=1,2 .
$$

This leads to $\gamma(r)=w_{2}^{\prime}+w_{1} w_{2}$, and $w_{1}(r)=\beta(r)-w_{2}(r)$, where $\beta(r)$ is given by (8), and $w_{2}$ has the form given in (14). Hence, $w_{1}(r)$ takes the form:

$$
w_{1}(r)=\frac{l}{r}-\frac{1}{l}-\frac{d}{d r} \ln \left[c_{1} g_{1}(r)+c_{2} g_{2}(r)\right] .
$$

It is now clear that when solving (13), we simultaneously have gotten the solutions to the equation $A^{\dagger} \widetilde{\psi}_{l-2, \epsilon}=0$, as well as the factorizations of the operator $A$. There is a continuous family of factorizations because when we change the values of $c_{1}$ and $c_{2}$ in (20), we are simultaneously changing the operators $b_{1}$ and $b_{2}$, but maintaining fixed their product $A=b_{2} b_{1}$.

A first consequence of this factorization arises after rewritting equation (2) as $\widetilde{H}_{l-2} b_{2} b_{1}=$ $b_{2} b_{1} H_{l}$. Suppose now that there is a Hamiltonian $H_{l-1}^{*}=-d^{2} / d r^{2}+V_{l-1}^{*}$ such that $H_{l-1}^{*} b_{1}=b_{1} H_{l}$. Thus $b_{2} H_{l-1}^{*} b_{1}=b_{2} b_{1} H_{l}$, and we get $\widetilde{H}_{l-2} b_{2}=b_{2} H_{l-1}^{*}$. Therefore, $H_{l-1}^{*}$ could be considered as an intermediate Hamiltonian between $H_{l}$, and $\widetilde{H}_{l-2}$. Hence, the SOIT can be seen as the iteration of two first order intertwinning transformations. 
We notice here that from the very beginning we are labeling with the subindex $l-1$ the intermediate Hamiltonian $H^{*}$. This is a consequence of the further calculations leading to a centrifugal term for $V^{*}$ with exactly that index.

In order to ensure that the first order intertwinning relationship $H_{l-1}^{*} b_{1}=b_{1} H_{l}$ would be satisfied, the functions $w_{1}$ and $V_{l-1}^{*}$ must satisfy some restrictions. The key one becomes the following Riccati equation:

$$
-w_{1}^{\prime}+\left(w_{1}\right)^{2}-V_{l}+\delta_{1}=0
$$

complemented with the typical SUSY relationship $V_{l-1}^{*}=V_{l}+2 w_{1}^{\prime}$, where $\delta_{1}$ is a constant to be determined. We notice also that a first order intertwinning relationship of the kind $H_{l-1}^{*} b_{1}=b_{1} H_{l}$ leads in a natural way to the factorization of the Hamiltonians $H_{l}$ and $H_{l-1}^{*}: H_{l}=b_{1}^{\dagger} b_{1}+\delta_{1}$, and $H_{l-1}^{*}=b_{1} b_{1}^{\dagger}+\delta_{1}$. By a similar argument, the Hamiltonians $H_{l-1}^{*}$ and $\widetilde{H}_{l-2}$ become factorized in terms of $b_{2}$ and $b_{2}^{\dagger}: H^{*}=b_{2}^{\dagger} b_{2}+\delta_{2}$, and $\widetilde{H}=b_{2} b_{2}^{\dagger}+\delta_{2}$.

In order to determine the intermediate Hamiltonian $H_{l-1}^{*}$, we must find inside the family (20) a member obeying equation (21). In fact, for $c_{1}=0$ and $c_{2}=1$, we have $\delta_{1}=-1 /(l-1)^{2}, l=2,3, \ldots$ Thus, the potential $V_{l-1}^{*}(r)$ can be written as:

$$
V_{l-1}^{*}(r)=\frac{l(l-1)}{r^{2}}-\frac{2}{r}+2\left[\frac{\left(g_{2}^{\prime}\right)^{2}-g_{2}^{\prime \prime} g_{2}}{\left(g_{2}\right)^{2}}\right] .
$$

The parameter domain for which the family (22) has the same singularity as $V_{l-1}(r)$ is given by $\nu_{2} \in(1, \infty)$. The eigenfunctions of $H_{l-1}^{*}, l=2,3, \ldots$ are given by $\psi_{l-1,-1}^{*} \propto$ $r^{l} e^{-r / l} / g_{2}$, and $\psi_{n, l-1}^{*} \propto b_{1} \psi_{n l}$, with eigenvalues $E_{l-1,-1}^{*}=-1 /(l-1)^{2}$, and $E_{l-1, k}^{*}=E_{l k}$, $k=1,2, \ldots$, respectively. Notice the unusual absence of the state corresponding to $E_{l-1,0}^{*}=$ $-1 / l^{2}$. A direct comparison of the spectra shows that $V_{l-1}^{*}(r)$ is almost isospectral to $V_{l-1}$, the difference resting on the ground state level position.

The next first order intertwinning transformation gives a different factorization of $H_{l-1}^{*}$ and some interesting new results. The absent energy level $\widetilde{E}_{l-2,0}=E_{l-1,0}^{*}$ is added now to the spectrum of $H_{l-1}^{*}$ in order to generate $\widetilde{H}_{l-2}$. But this means that the factorization energy in this second step is greater than the ground state energy level of $H_{l-1}^{*}$, and this naturally fills the hole generated by the first factorization. The eigenfunctions of $\widetilde{H}_{l-2}$, in terms of those of $H_{l-1}^{*}, l=2,3, \ldots$, are given by $\left\{\widetilde{\psi}_{l-2,-1} \propto b_{2} \psi_{l-1,-1}^{*}, \widetilde{\psi}_{l-2,0}, \widetilde{\psi}_{n, l-2} \propto\right.$ $\left.b_{2} \psi_{n, l-1}^{*}\right\}$, where $\widetilde{\psi}_{l-2,0} \propto \exp \left(-\int w_{2}(x) d x\right)$ is the eigenfunction associated to the 'missing' energy level $E_{l-1,0}^{*}=\widetilde{E}_{l-2,0}$. A direct calculation shows that this set of eigenfunctions is the same as that derived by means of the SOIT.

Concluding Remarks. In this paper we have shown that the Second Order Intertwinning Technique allows one to derive a two-parametric family of isospectral Hydrogenlike potentials. The iteration of two first order intertwinning transformations leads to the 
same results but gives additional information. Thus, against the standard statement of SUSY QM, there are cases where a factorization energy greater than the ground state energy of the departure Hamiltonian leads to a physically acceptable SUSY partner. A deeper discussion of these first order intertwinning cases will be given elsewhere [13]. Finally, all the potentials presented here have the same kind of singularity at a fixed point $(r=0)$ as the initial Hydrogen-like Hamiltonian. The case when the intertwined potentials are non-singular has been illustrated by Fernández et.al. for the harmonic oscillator [6, 10, 12], while the case when the SUSY partner of the oscillator potential has a movable singularity has been successfully interpreted [14]. The corresponding problem for a family of isospectral Hydrogen-like potentials with a different singularity as those of $V_{l}(r)$ in $r=0$ is open.

\section{Acknowledgements}

This work is supported by a Postdoctoral CONACyT fellowship (México) in the program

"Programa de Estancias Posdoctorales en Instituciones del extranjero 1997-1998". The author is indebted to Dr. D. J. Fernández for enlightenning discussions and suggestions. 


\section{References}

[1] G. Darboux, Compt. Rend. Acad. Sci. 94, 1456 (1882).

[2] I.M. Gelfand and B.M. Levitan, Am. Math. Soc. Trans. 1, 253 (1951).

[3] E. Schrödinger, Proc. Roy. Irish Acad. 46 A, 9 (1940). I. Infeld and T.E. Hull, Rev. Mod. Phys. 23, 21 (1951)

[4] B. Mielnik, J. Math. Phys.25, 3387 (1984).

[5] E. Witten, Nucl. Phys. B 188, 513 (1981); B 202, 253 (1982). F. Cooper, A. Khare, U. Sukhatme, Phys. Rep. 251, 267 (1995). J. Beckers, N. Debergh and C. Gotti, Helvetica Phys. Acta 71, 214 (1998)

[6] R.W. Carrol, Transmutation and Operator Differential Equations. North-Holland Mathematics Studies 37, The Netherlands (1979).

D.J. Fernández, Int. J. Mod. Phys. A 12, 171 (1997).

[7] P.B. Abraham and H.E. Moses, Phys. Rev. A22, 1333 (1980). A. Andrianov and N.V. Borisov, Teor. Math. Phys. 61, 1078 (1985). B.F. Samsonov, J. Phys. A 28, 6989 (1995). V.G. Bagrov and B.F. Samsonov, J. Phys. A 29, 1011 (1996).

[8] D.J. Fernández, Lett. Math. Phys. 8, 337 (1984).

[9] D.J. Fernández, J. Negro and Mariano A. del Olmo, Ann. Phys. 252, 386 (1996). V.M. Eleonsky and V.G. Korelev, Phys. Rev. 55, 2580 (1997). G. Junker and P. Roy, Supersymmetric construction of exactly solvable potentials and non-linear algebras, PREPRINT quant-ph/9709021

[10] D.J. Fernández, L.M. Glasser and L.M. Nieto, New Isospectral Oscillator Potentials, to appear in Phys. Letts A

[11] A.A. Andrianov, M.V. Ioffe and V.P. Spiridonov, Phys. Letts A, 174, 273 (1993). A.A. Andrianov, M.V. Ioffe, F. Cannata and J.P. Dedonder, Int. J. Mod. Phys. A 10, 2683 (1995).

[12] D.J. Fernández, V. Hussin and B. Mielnik, A simple generation of exactly solvable anharmonic oscillators, PREPRINT CINVESTAV, México. (1998). 
[13] J.O. Rosas-Ortiz, Ph.D. Thesis, CINVESTAV-IPN, México (1997).

J.O. Rosas-Ortiz, Exactly Solvable Hydrogen-like Potentials and Factorization Method, in preparation.

[14] I.F. Márquez, J. Negro and L.M. Nieto, Factorization method and singular Hamiltonians, to appear in J. Phys. A. 


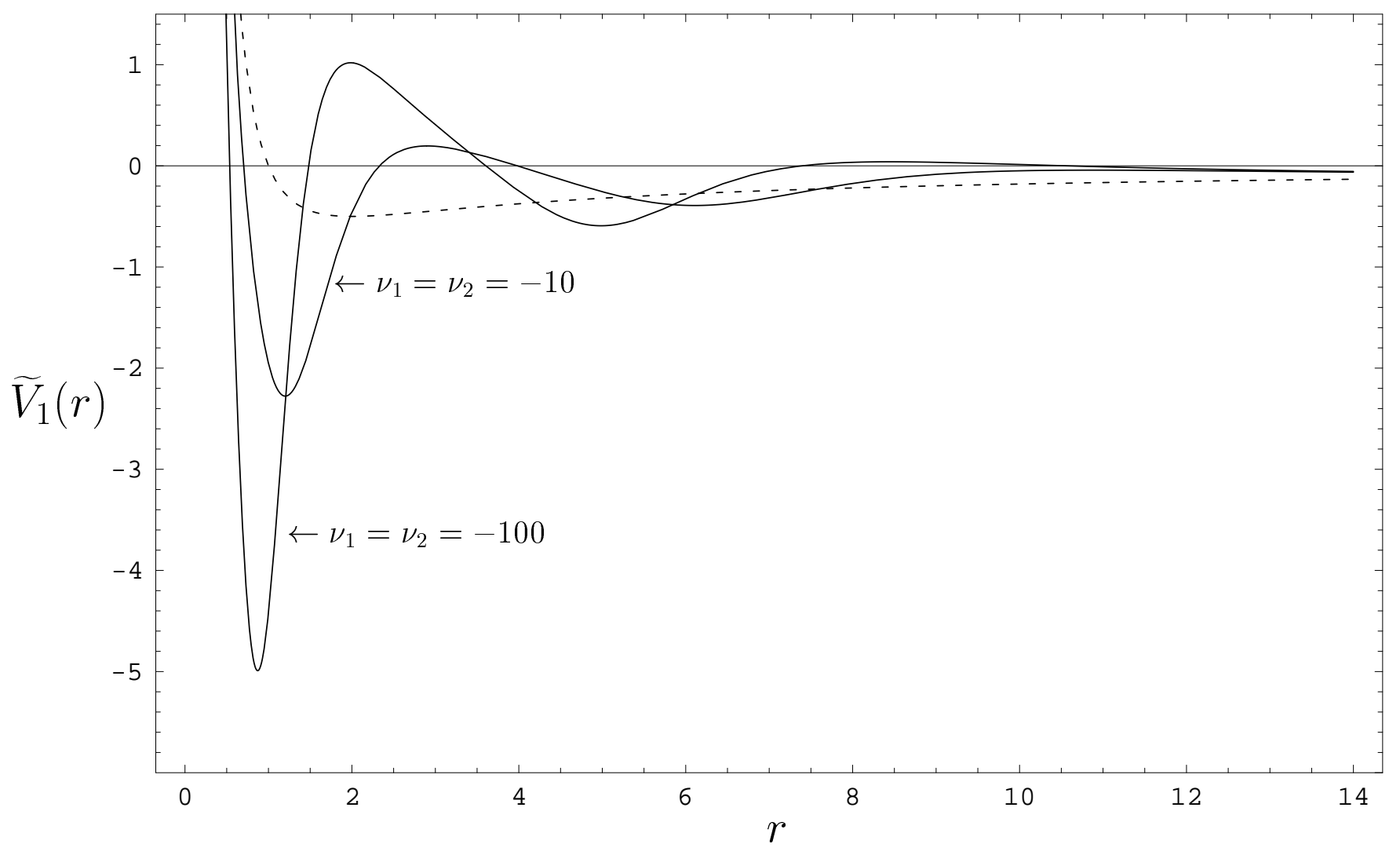

Figure 1: The Hydrogen-like potential $V_{l-2}(r)$ (dashed line) and two members of the family $\widetilde{V}_{l-2}(r)$ (solid line) with $l=3$. Here we have chosen equal values for $\nu_{1}$ and $\nu_{2}$, but it is not a restriction (see eq 11 and bellow). Notice that in the limit case when $\nu_{i} \rightarrow 0$, $i=1,2$, the solid lines resemble more and more the dashed one. 


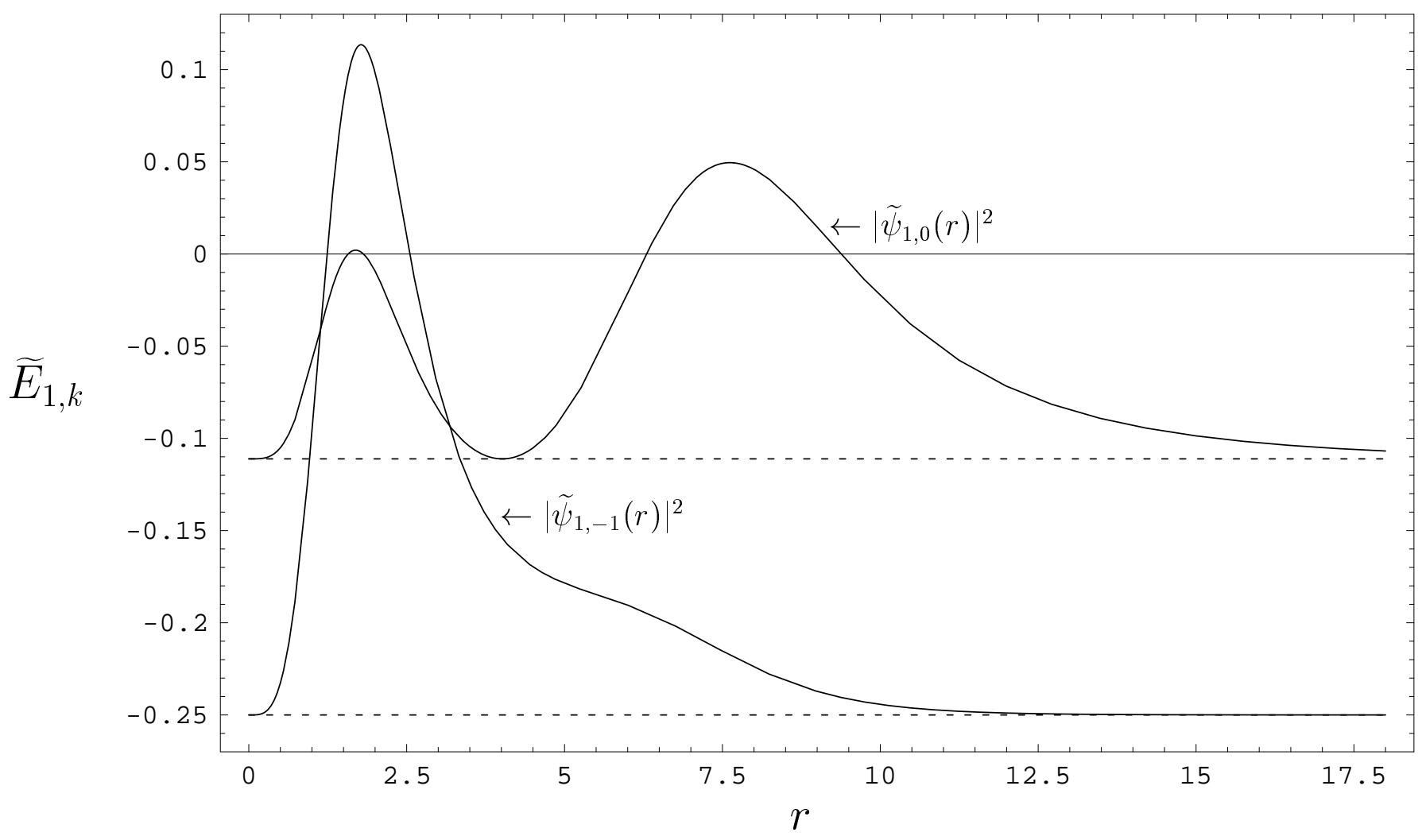

Figure 2: Behaviour of the probability densities of the two first energy levels $-1 / 4$ and $-1 / 9$ of $\widetilde{H}_{1}$, with $\nu_{1}=\nu_{2}=-10$. The levels are indicated by the dashed lines 